\title{
Model cell systems representing expression regulation and signal transduction of thymic stromal lymphopoietin (TSLP) in the development of asthmatic symptoms
} A Wohlmann*, K Sebastian, A Borowski, S Krause and K Friedrich

Address: Friedrich-Schiller-Universität Jena, Institute of Biochemistry, Jena, Gemany

* Corresponding author

from 12th Joint Meeting of the Signal Transduction Society (STS). Signal Transduction: Receptors, Mediators and Genes

Weimar, Germany. 29-31 October 2008

Published: 26 February 2009

Cell Communication and Signaling 2009, 7(SuppI I):A93 doi:I0.II86/I478-8IIX-7-SI-A93

This abstract is available from: http://www.biosignaling.com/content/7/SI/A93

(c) 2009 Wohlmann et al; licensee BioMed Central Ltd.

Thymic stromal lymphopoietin (TSLP) is a novel interleukin-7-like cytokine, which triggers dendritic cell-mediated inflammatory responses ultimately executed by Th2 helper cells. TSLP is a central player in the development of allergic, especially asthmatic symptoms, and has been suggested to represent a missing link in the information flow from antigen-exposed epithelial cells via dendritic cells (DCs) to T helper cells.

We have challenged the lung epithelial cell line A549 with various allergens such as extracts from dust mites, pollen and fungi, and could show by real time PCR for the first time an allergen-dependent specific transcriptional upregulation of TSLP.

To approach the as yet poorly defined TSLP-induced signal transduction in dendritic cells, we established a cellular model system on the basis of the murine pro- $\mathrm{B}$ cell line $\mathrm{Ba} / \mathrm{F} 3$. The heterodimeric human TSLP receptor (hTSLPR) consisting of the novel TSLP receptor chain and the IL-7 receptor alpha chain was functionally reconstituted in factor-dependent $\mathrm{Ba} / \mathrm{F} 3$ cells. We could demonstrate by phosphotyrosine protein analysis and by STAT type-specific reporter gene assays, that the ligand-stimulated TSLPR triggers activation of STAT3 and STAT5 as well as of STAT1. Employment of specific inhibitors proved the functional involvement of Janus kinases (JAKs) in TSLPdependent, receptor-mediated cellular responses.
These achievements provide tools for research towards a better molecular understandimg of atopic asthma and for the future development of new therapeutics interfering with TSLP function. 